Supporting Information

\title{
Synthesis, characterization of a crown-shaped 36-molybdate cluster and application in catalyzing Knoevenagel condensation
}

Qiaofei Xu, Baijie Xu, Hui Kong, Peipei He, Jiawei Wang, Thirumurthy Kannan, Pengtao Ma, Jingping Wang* and Jingyang Niu*

Henan Key Laboratory of Polyoxometalate Chemistry, Institute of Molecule and Crystal Engineering, College of Chemistry and Chemical Engineering, Henan University, Kaifeng, Henan 475004, P. R. China.E-mail:jyniu@henu.edu.cn; jpwang@henu.edu.cn.

CONTAINS:

1. Additional Structural Figures

2. Characterization

3. Catalytic experiments in detail.

4. Mass spectrum for the corresponding product.

5. Catalytic device

6. Scale-up experiment 


\section{Additional Structural Figures}
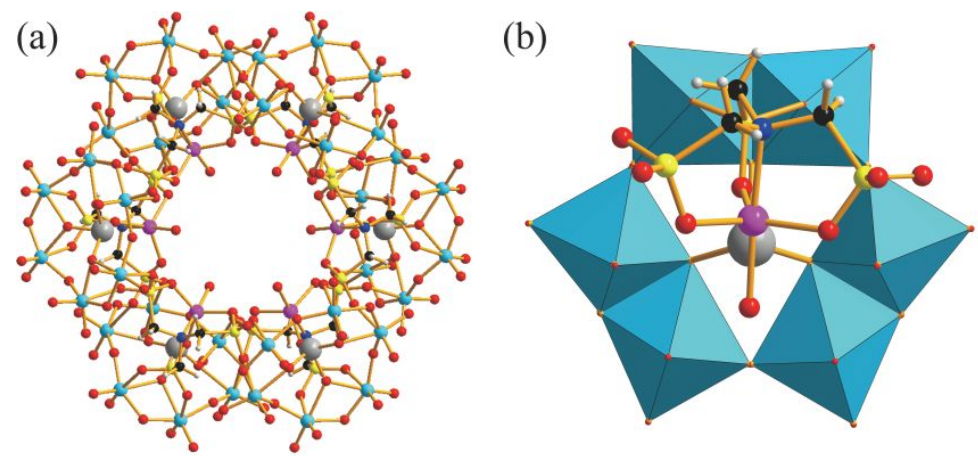

(c)

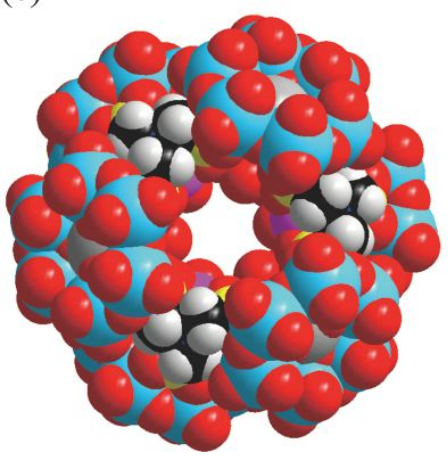

(d)

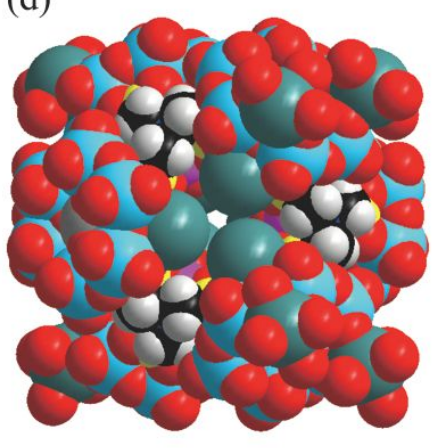

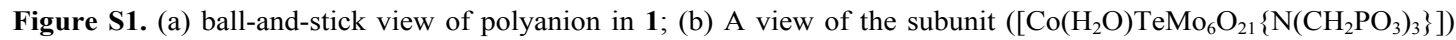
viewed down the pseudo 3-fold axis; (c) space-filling view of polyanion in 1; (d) space-filling view of $\mathbf{1}$. $\left(\mathrm{MoO}_{6} / \mathrm{Mo}\right.$ : sky blue, Te: gray-50\%, P: yellow, Co: cobalt, C: black, N: blue, O: red, H: gray-25\%)

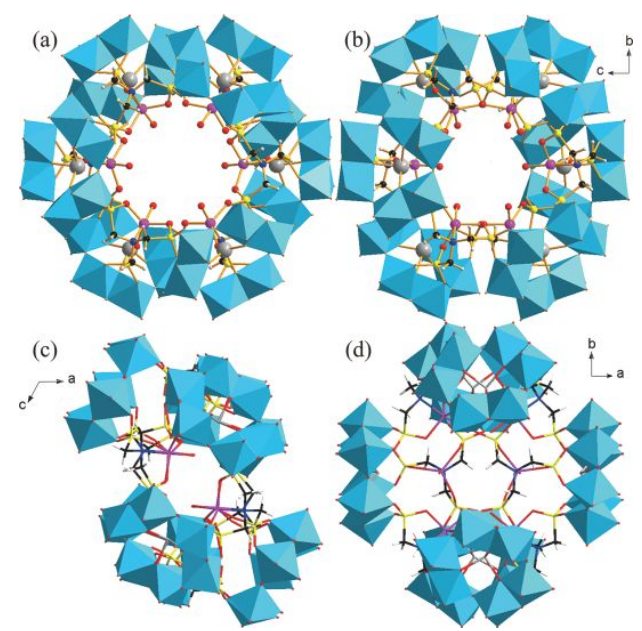

Figure S2. (a) polyhedral / ball-and-stick view of the polyanion of 1; The view of the polyanion of 1 from different sides: (b) view along axis of a; view along axis of b; (c) view along axis of c. $\left(\mathrm{MoO}_{6} / \mathrm{Mo}\right.$ : sky blue, Te: gray-50\%, P: yellow, Co: cobalt, C: black, N: blue, O: red, H: gray-25\%) 

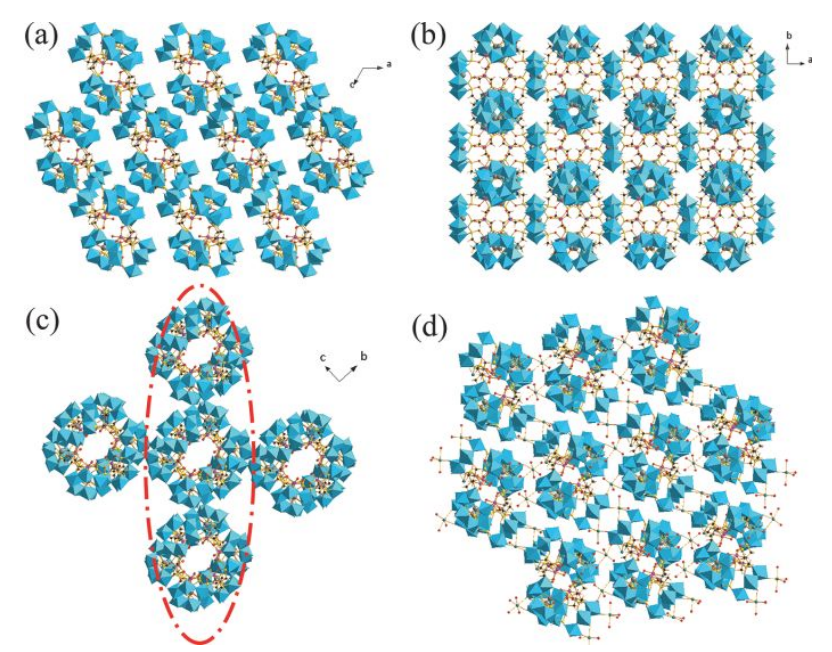

Figure S3. The stacking diagram of the polyanion in 1 from different view: (a) view along axis of b; (b) view along axis of c; (c) view along axis of $\mathrm{a}$; (d) the unfolded view of the $2 \mathrm{D}$ plane in the stacking diagram.

\section{Characterization}

\section{IR spectrum}

The infrared spectrum of $\mathbf{1}$ in Figure S4 shows the skeletal vibrations in the region between 500 and 4000 $\mathrm{cm}^{-1}$, which is in good agreement with the result of single-crystal X-ray structural analysis. Here, the terminal $\mathrm{Mo}-\mathrm{O}_{\mathrm{t}}$ vibrations are seen as prominent bands at 934 and $893 \mathrm{~cm}^{-1}$ and the peaks in the range of 779-647 $\mathrm{cm}^{-1}$ are assigned to Mo-O-Mo bridges vibrations. The characteristic peaks at 1067 and 1101 $\mathrm{cm}^{-1}$ are derived from $\mathrm{P}-\mathrm{O}$ vibration. The peaks in 3166 and $1400 \mathrm{~cm}^{-1}$ are ascribed to the stretching vibration and bend vibration of $\mathrm{N}-\mathrm{H}$ bond of $\mathrm{NH}_{4}{ }^{+}$. In addition, the strong band at $3430 \mathrm{~cm}^{-1}$ corresponds to the stretching vibration of $\mathrm{O}-\mathrm{H}$ and the sharp peak at $1640 \mathrm{~cm}^{-1}$ corresponds to the bend vibration of $\mathrm{O}-\mathrm{H}$ in crystal water.

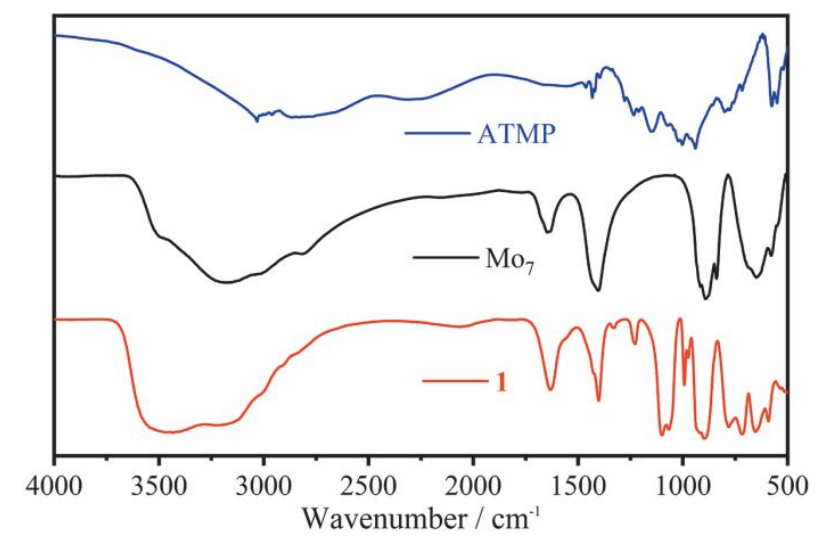

Figure S4. (a) The contrastive infrared spectrum of $\mathbf{1}$ (red line), $\mathrm{Mo}_{7}$ (black line) and ATMP (blue line); 


\section{XPRD}

In order to detect the purity of test sample, the powder XRD analysis of $\mathbf{1}$ has completed. In Figure S5, the powder XRD pattern of $\mathbf{1}$ is in agreement with its simulated one from X-ray single-crystal data, and peak intensity is discrepant due to the anisotropic effects of crystal, implying that the sample used for all the characterization and test is pure.

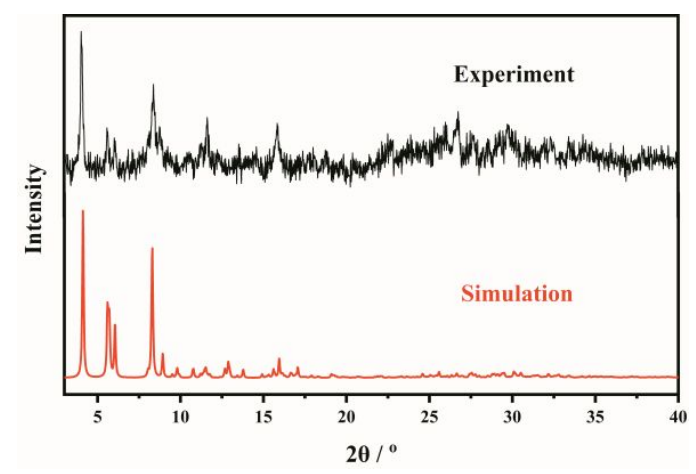

Figure S5. The powder XRD pattern of 1.

\section{TG analysis}

The thermal behavior of 1 has been analyzed upon heating to $800{ }^{\circ} \mathrm{C}$ (Figure S6). The TGA curve shows one successive mass losses between 25 and $850{ }^{\circ} \mathrm{C}$, which indicated that all the water molecules and $\mathrm{NH}_{4}{ }^{+}$escaped from the structure without obvious demarcation temperature. Hence, $8.4 \%$ of mass loss was assigned to 42 lattice water (cal. $7.8 \%$ ). From 100 to $140{ }^{\circ} \mathrm{C}$, the weight loss $(2.42 \%)$ can be attributed to 12 water molecules in the structure (cal. 2.23\%).

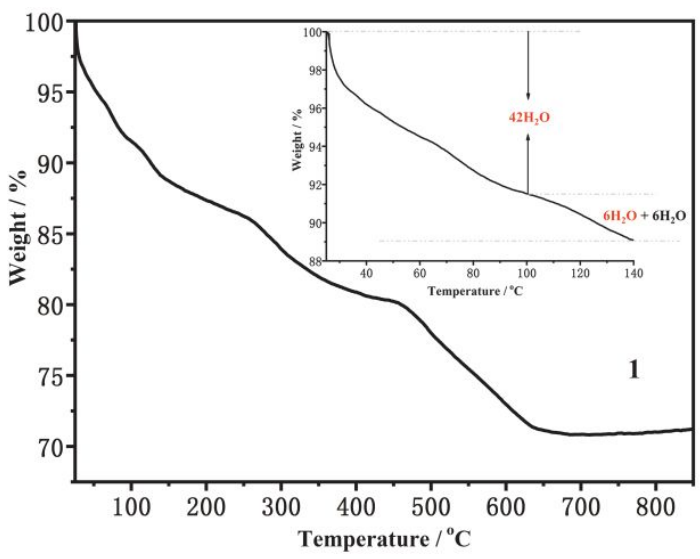

Figure S6. Thermogravimetric analysis (TGA) curve of 1

\section{X-ray photoelectron spectroscopy (XPS)}




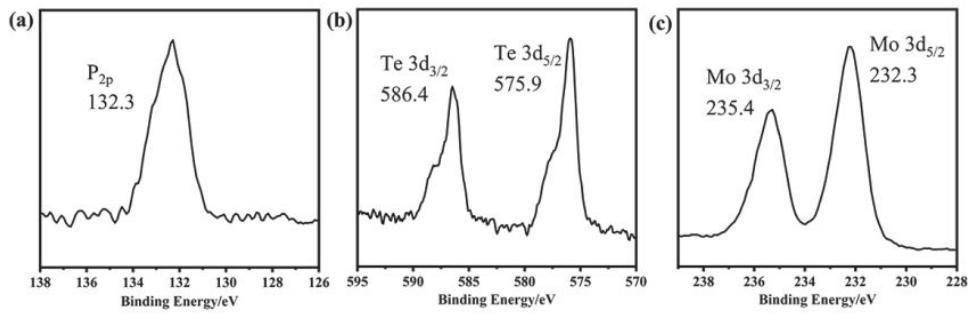

Figure S7. XPS spectrum of the sample: (a) P 2p; (b) Te 3d; (c) Mo 3d.

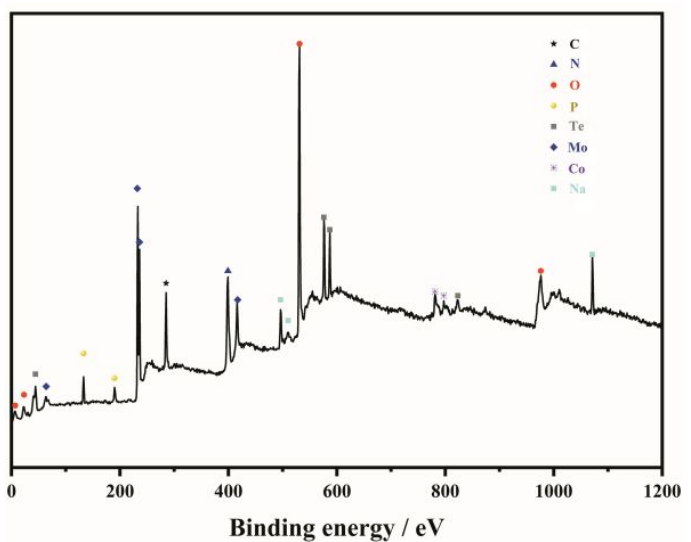

Figure S8. wide-scan X-ray photoelectron spectra of the sample

Table S1. Selected elemental composition by XPS analysis for $\mathbf{1}$.

\begin{tabular}{cccccc}
\hline $\begin{array}{c}\mathrm{C} \\
(\text { at.\% })\end{array}$ & $\begin{array}{c}\mathrm{N} \\
(\text { at.\% })\end{array}$ & $\begin{array}{c}\mathrm{P} \\
\text { (at.\%) }\end{array}$ & $\begin{array}{c}\mathrm{Te} \\
\text { (at.\%) }\end{array}$ & $\begin{array}{c}\text { Mo } \\
\text { (at.\%) }\end{array}$ & $\begin{array}{c}\text { Co } \\
\text { (at.\%) }\end{array}$ \\
\hline \multirow{2}{*}{17.66} & 22.25 & 17.23 & 5.23 & 32.42 & 5.21 \\
\hline
\end{tabular}

\section{Bond valence calculation of $P$ atoms and Te atoms}

Table S2. the Bond valence calculation of $\mathrm{P}$ atoms

\begin{tabular}{llllll}
\hline Bond & Bond length & Bond Valence & Bond & Bond length & Bond Valence \\
\hline $\mathrm{P} 1-\mathrm{O} 58$ & 1.519 & 1.350 & $\mathrm{P} 2-\mathrm{O} 46$ & 1.542 & 1.269 \\
$\mathrm{P} 1-\mathrm{O} 59$ & 1.540 & 1.275 & $\mathrm{P} 2-\mathrm{O} 67$ & 1.554 & 1.228 \\
$\mathrm{P} 1-\mathrm{O} 76$ & 1.517 & 1.357 & $\mathrm{P} 2-\mathrm{O} 81$ & 1.538 & 1.282 \\
$\mathrm{P} 1-\mathrm{C} 1$ & 1.824 & $\mathrm{P} 2-\mathrm{C} 2$ & 1.851 & 1.111 \\
\hline \multicolumn{5}{c}{$\sum(\mathrm{P} 2)=2.668$} \\
\hline
\end{tabular}




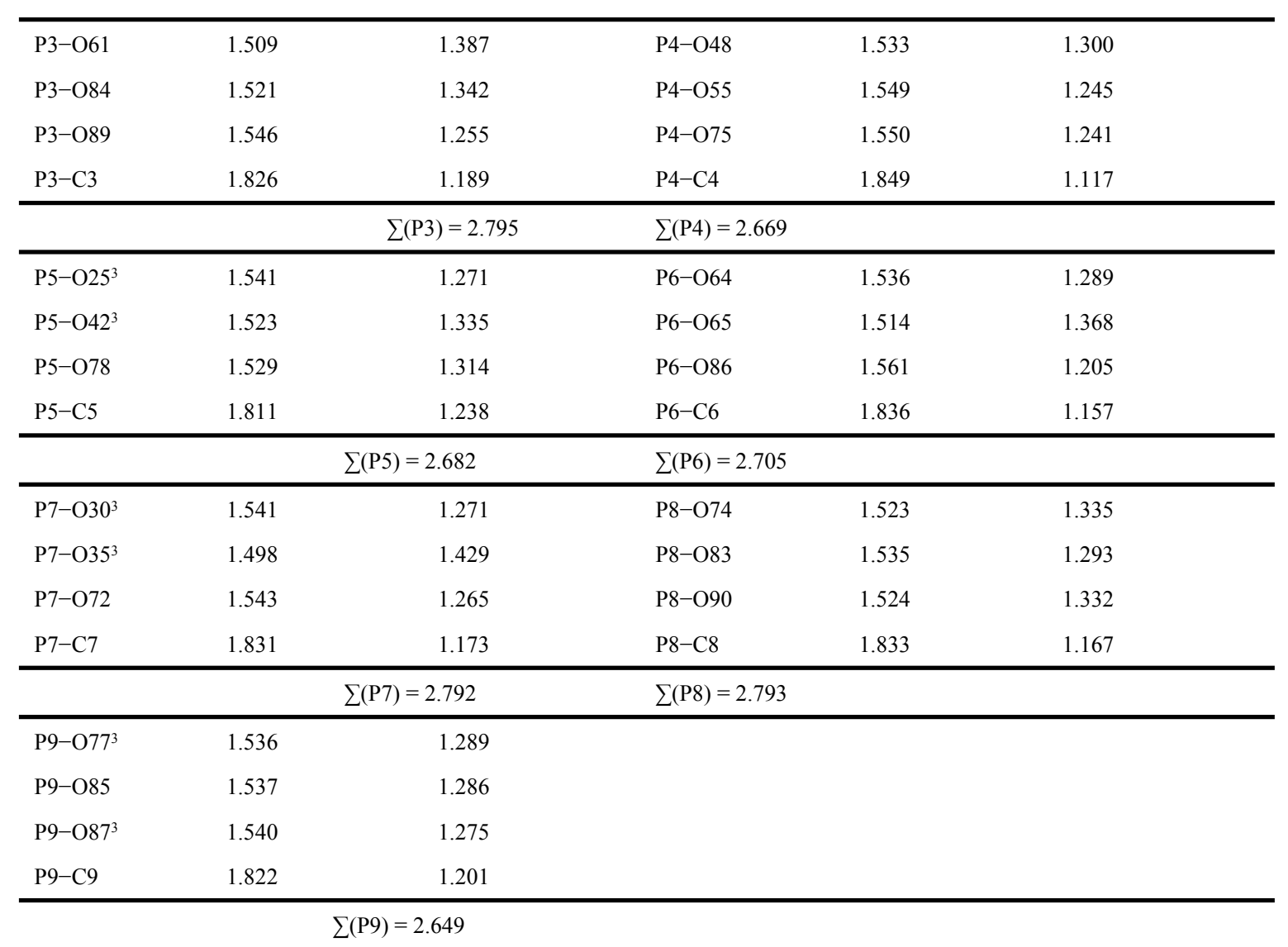

Table S3. the Bond valence calculation of Te atoms

\begin{tabular}{|c|c|c|c|c|c|}
\hline Bond & Bond length & Bond Valence & Bond & Bond length & Bond Valence \\
\hline Te1-O62 & 1.886 & 1.279 & $\mathrm{Te} 2-\mathrm{O} 50$ & 1.879 & 1.303 \\
\hline Te1-O79 & 1.903 & 1.221 & $\mathrm{Te} 2-\mathrm{O} 52$ & 1.883 & 1.289 \\
\hline Te1-O82 & 1.880 & 1.300 & $\mathrm{Te} 2-\mathrm{O} 71$ & 1.856 & 1.387 \\
\hline \multicolumn{4}{|c|}{$\sum(\mathrm{Te} 1)=3.800$} & \multicolumn{2}{|c|}{$\sum(\mathrm{Te} 2)=3.979$} \\
\hline Te3-O41 & 1.878 & 1.307 & & & \\
\hline Te3-O43 & 1.896 & 1.245 & & & \\
\hline Te3-O54 & 1.894 & 1.251 & & & \\
\hline \multicolumn{6}{|c|}{$\sum(\mathrm{Te} 3)=3.803$} \\
\hline
\end{tabular}

Table S4. the Bond valence calculation of $\mathrm{O}$ atoms

\begin{tabular}{|c|c|c|c|c|c|c|c|}
\hline Atom 1 & Atom 2 & Bond length & $\begin{array}{c}\text { Bond Valence } \\
\left(\sum(O)\right)\end{array}$ & Atom 1 & Atom 2 & Bond length & $\begin{array}{c}\text { Bond Valence } \\
(\Sigma(O))\end{array}$ \\
\hline $\mathrm{O} 1$ & Mo13 & 1.71 & 1.70 & \multirow{2}{*}{ O54 } & Mo9 & 1.90 & \multirow{2}{*}{1.91} \\
\hline $\mathrm{O} 2$ & Mo10 & 1.68 & 1.85 & & Mo10 & 1.95 & \\
\hline $\mathrm{O} 3$ & Mo18 & 1.69 & 1.80 & \multirow{2}{*}{ O55 } & P3 & 1.54 & \multirow{2}{*}{1.62} \\
\hline $\mathrm{O} 4$ & Mo16 & 1.71 & 1.70 & & Mo15 & 2.22 & \\
\hline
\end{tabular}




\begin{tabular}{|c|c|c|c|c|c|c|c|}
\hline O5 & Mo13 & 1.71 & 1.70 & \multirow{2}{*}{ O56 } & Mo12 & 1.91 & \multirow{2}{*}{1.93} \\
\hline O6 & Mo11 & 1.69 & 1.80 & & Mo11 & 1.93 & \\
\hline O7 & Mo18 & 1.70 & 1.75 & \multirow{2}{*}{ O57 } & $\mathrm{P} 3$ & 1.51 & \multirow{2}{*}{1.63} \\
\hline $\mathrm{O} 8$ & Mo11 & 1.70 & 1.75 & & Mo16 & 2.31 & \\
\hline O9 & Mo10 & 1.72 & 1.66 & \multirow{2}{*}{ O58 } & Mo7 & 1.89 & \multirow{2}{*}{2.01} \\
\hline $\mathrm{O} 10$ & Mo15 & 1.72 & 1.66 & & Mo12 & 1.92 & \\
\hline O11 & Mo15 & 1.70 & 1.75 & \multirow{2}{*}{ O59 } & P6 & 1.54 & \multirow{2}{*}{1.45} \\
\hline O12 & Mo16 & 1.66 & 1.95 & & $\mathrm{Cu} 2$ & 2.17 & \\
\hline $\mathrm{O} 13$ & Mo6 & 1.68 & 1.85 & \multirow{2}{*}{ O60 } & P4 & 1.52 & \multirow{2}{*}{1.67} \\
\hline \multirow{2}{*}{ O14 } & Mo14 & 1.72 & \multirow{2}{*}{1.68} & & Mo12 & 2.23 & \\
\hline & $\mathrm{H} 4 \mathrm{~B}$ & 2.37 & & \multirow{2}{*}{ O61 } & P5 & 1.52 & \multirow{2}{*}{1.66} \\
\hline O15 & Mo5 & 1.72 & 1.66 & & $\mathrm{Cu} 2$ & 2.01 & \\
\hline O16 & Mo17 & 1.69 & 1.80 & \multirow{2}{*}{ O62 } & $\mathrm{P} 4$ & 1.52 & \multirow{2}{*}{1.70} \\
\hline O17 & Mo17 & 1.71 & 1.70 & & $\mathrm{Cu} 2$ & 1.98 & \\
\hline $\mathrm{O} 18$ & Mo9 & 1.69 & 1.80 & \multirow{2}{*}{ O63 } & P3 & 1.51 & \multirow{2}{*}{1.76} \\
\hline \multirow{2}{*}{ O19 } & Mo9 & 1.72 & \multirow{2}{*}{1.86} & & $\mathrm{Cu} 1$ & 1.96 & \\
\hline & $\mathrm{Na} 2$ & 2.40 & & \multirow{3}{*}{ O64 } & $\mathrm{Te} 2$ & 1.87 & \multirow{3}{*}{2.14} \\
\hline $\mathrm{O} 20$ & Mo14 & 1.70 & 1.75 & & Mo10 & 2.20 & \\
\hline $\mathrm{O} 21$ & Mo5 & 1.68 & 1.85 & & Mo9 & 2.29 & \\
\hline $\mathrm{O} 22$ & Mo1 & 1.71 & 1.70 & & Mo4 & 1.90 & \multirow{2}{*}{1.91} \\
\hline \multirow{2}{*}{$\mathrm{O} 23$} & Mo6 & 1.70 & \multirow{2}{*}{1.77} & 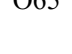 & Mo5 & 1.95 & \\
\hline & H9B & 2.48 & & & $\mathrm{Te} 1$ & 1.87 & \\
\hline $\mathrm{O} 24$ & Mo12 & 1.68 & 1.85 & O66 & Mo4 & 2.21 & 2.17 \\
\hline 025 & Mo13 & 1.90 & 10 & & Mo5 & 2.25 & \\
\hline 023 & Mo14 & 1.97 & 1.00 & & Mo1 & 1.90 & \\
\hline & Mo18 & 1.93 & & 801 & Mo6 & 1.94 & 10.0 \\
\hline & Mo17 & 1.96 & & Oro & Mo5 & 1.89 & \\
\hline $\mathrm{O} 27$ & Mo4 & 1.70 & 1.75 & 000 & Mo6 & 1.91 & 2.04 \\
\hline & Mo15 & 1.91 & & & Mo9 & 1.89 & \\
\hline 020 & Mo16 & 1.95 & 1.00 & 009 & Mo8 & 1.91 & 2.04 \\
\hline 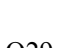 & Mo12 & 1.72 & 10 & & P6 & 1.52 & \\
\hline 823 & $\mathrm{Na} 2$ & 2.41 & & O70 & Mo14 & 2.22 & 1.70 \\
\hline $\mathrm{O} 30$ & Mo8 & 1.69 & 1.80 & & $\mathrm{H} 4 \mathrm{~B}$ & 2.43 & \\
\hline & Mo1 & 1.71 & & & P7 & 1.53 & \\
\hline 031 & $\mathrm{Na} 1$ & 2.41 & 1.90 & $0 / 1$ & $\mathrm{Cu} 3$ & 2.02 & 1.02 \\
\hline 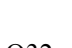 & Mo7 & 1.71 & & & P8 & 1.50 & \\
\hline 032 & $\mathrm{Na} 2$ & 2.45 & 1.88 & $0 / 2$ & Mo1 & 2.29 & 1.08 \\
\hline 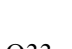 & Mo7 & 1.69 & 10 & 070 & P8 & 1.51 & \\
\hline ט & $\mathrm{H} 3 \mathrm{~B}$ & 2.47 & 1.01 & 2013 & $\mathrm{Cu} 3$ & 2.15 & 1.0 \\
\hline 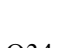 & Mo18 & 1.90 & O & & P2 & 1.51 & \\
\hline 034 & Mo13 & 1.91 & 2.01 & 014 & $\mathrm{Cu} 1$ & 2.18 & 1.03 \\
\hline
\end{tabular}




\begin{tabular}{|c|c|c|c|c|c|c|c|}
\hline O35 & Mo2 & 1.69 & 1.80 & \multirow{3}{*}{ O75 } & P2 & 1.50 & \multirow{3}{*}{1.76} \\
\hline \multirow{2}{*}{ O36 } & Mo8 & 1.69 & \multirow{2}{*}{1.98} & & Mo7 & 2.23 & \\
\hline & $\mathrm{Na} 2$ & 2.43 & & & H3B & 2.48 & \\
\hline \multirow{2}{*}{$\mathrm{O} 37$} & Mo4 & 1.71 & \multirow{2}{*}{1.89} & \multirow{3}{*}{ O76 } & $\mathrm{P} 2$ & 1.50 & \multirow{3}{*}{1.69} \\
\hline & $\mathrm{Na} 1$ & 2.42 & & & Mo8 & 2.28 & \\
\hline $\mathrm{O} 38$ & Mo3 & 1.71 & 1.70 & & $\mathrm{Na} 4$ & 2.49 & \\
\hline \multirow{2}{*}{ O39 } & Mo10 & 1.90 & \multirow{2}{*}{2.04} & \multirow{3}{*}{ O77 } & $\mathrm{Te} 1$ & 1.88 & \multirow{3}{*}{2.12} \\
\hline & Mo11 & 1.90 & & & Mo3 & 2.19 & \\
\hline \multirow{2}{*}{$\mathrm{O} 40$} & P5 & 1.51 & \multirow{2}{*}{1.64} & & Mo2 & 2.29 & \\
\hline & Mo10 & 2.29 & & \multirow{2}{*}{ O78 } & P9 & 1.52 & \multirow{2}{*}{1.66} \\
\hline \multirow{2}{*}{ O41 } & Mo2 & 1.71 & \multirow{2}{*}{1.88} & & Mo5 & 2.24 & \\
\hline & $\mathrm{Na} 1$ & 2.44 & & \multirow{3}{*}{ O79 } & $\mathrm{Te} 1$ & 1.87 & \multirow{3}{*}{2.15} \\
\hline \multirow{3}{*}{$\mathrm{O} 42$} & P7 & 1.50 & \multirow{3}{*}{1.88} & & Mo6 & 2.23 & \\
\hline & Mo18 & 2.30 & & & Mo1 & 2.25 & \\
\hline & $\mathrm{Na} 3$ & 2.37 & & \multirow{2}{*}{ O80 } & P9 & 1.50 & \multirow{2}{*}{1.64} \\
\hline 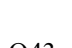 & Mo2 & 1.92 & & & Mo4 & 2.33 & \\
\hline 043 & Mo3 & 1.94 & 1.00 & & $\mathrm{P} 1$ & 1.51 & \\
\hline $\mathrm{OA}$ & P4 & 1.51 & 10 & 001 & Mo2 & 2.27 & 1.00 \\
\hline 044 & Mo11 & 2.32 & 1.02 & & Mo8 & 1.90 & \\
\hline & Mo14 & 1.90 & & 082 & Mo7 & 1.96 & 1.0 \\
\hline $0+3$ & Mo15 & 1.91 & 2.01 & & P7 & 1.52 & \\
\hline & P6 & 1.52 & & 800 & Mo17 & 2.27 & $100 \mathrm{~J}$ \\
\hline O46 & Mo13 & 2.27 & 1.80 & & $\mathrm{P} 1$ & 1.53 & \\
\hline & $\mathrm{Na} 3$ & 2.46 & & 084 & $\mathrm{Cu} 1$ & 1.98 & 1.00 \\
\hline & $\mathrm{Te} 3$ & 1.86 & & & Mo2 & 1.89 & \\
\hline O47 & Mo15 & 2.24 & 2.19 & 000 & Mo1 & 1.92 & 2.01 \\
\hline & Mo16 & 2.24 & & & P8 & 1.49 & \\
\hline & $\mathrm{Te} 2$ & 1.86 & & O86 & Mo6 & 2.25 & 1.77 \\
\hline O48 & Mo11 & 2.22 & 2.20 & & H9B & 2.45 & \\
\hline & Mo12 & 2.25 & & & Mo3 & 1.89 & 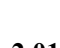 \\
\hline & $\mathrm{Te} 3$ & 1.87 & & 081 & Mo4 & 1.92 & 2.01 \\
\hline O49 & Mo14 & 2.22 & 2.17 & & $\mathrm{Te} 2$ & 1.87 & \\
\hline & Mo13 & 2.24 & & O88 & Mo7 & 2.24 & 2.14 \\
\hline & P5 & 1.50 & & & Mo8 & 2.25 & \\
\hline O50 & Mo9 & 2.27 & 1.87 & ( & P9 & 1.52 & \\
\hline & $\mathrm{Na} 4$ & 2.46 & & 089 & $\mathrm{Cu} 3$ & 1.99 & 1.09 \\
\hline & $\mathrm{Te} 3$ & 1.87 & & & P1 & 1.51 & \\
\hline O51 & Mo17 & 2.20 & 2.15 & 090 & Mo3 & 2.26 & $1.6 \%$ \\
\hline & Mo18 & 2.28 & & O91 & $\mathrm{Cu} 1$ & 1.96 & 0.47 \\
\hline$-\pi$ & Mo17 & 1.89 & ? & O92 & $\mathrm{Cu} 2$ & 1.98 & 0.44 \\
\hline USL & Mo16 & 1.91 & 2.04 & O93 & $\mathrm{Cu} 3$ & 1.96 & 0.47 \\
\hline
\end{tabular}




\begin{tabular}{|l|l|l|l|l|l|l|l|}
\hline \multirow{2}{*}{ O53 } & Mo3 & 1.71 & \multirow{2}{*}{$\mathbf{1 . 9 1}$} & & & & \\
\cline { 2 - 5 } & Na1 & 2.39 & & & & \\
\hline
\end{tabular}

\section{Catalytic experiments}

Table S5. Knoevenagel condensation of benzaldehyde with ethyl cyanoacetate catalyzed by different catalysts. ${ }^{a}$

\begin{tabular}{lllll}
\hline Entry & Catalyst & Conv. ${ }^{b}[\%]$ & Yield [\%] & Reaction system \\
\hline 1 & -- & 3 & 3 & -- \\
2 & $\mathrm{CoCl}_{2}$ & 8 & 8 & \\
3 & $\mathrm{ATMP}$ & 2 & 2 & Homogeneous \\
4 & $\mathrm{Mo}_{7}$ & 74 & 73 & \\
$5^{c}$ & $\mathrm{Mo}_{7}$ & 90 & 89 & \\
6 & $\mathrm{Na}_{2} \mathrm{TeO}_{3}$ & 62 & 60 & Heterogeneous \\
7 & $\mathbf{1}$ & 88 & 88 & \\
\hline
\end{tabular}

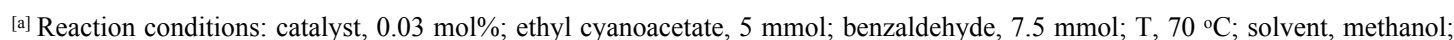
time, $2 \mathrm{~h}$. ${ }^{[b]} \mathrm{GC}$ yields for target product were based on methylbenzene as internal standard. ${ }^{c} \mathrm{Mo}_{7}, 0.16 \mathrm{~mol} \%$; All of the products were identified by GC-MS spectra and GC spectra.

\section{Recyclability}
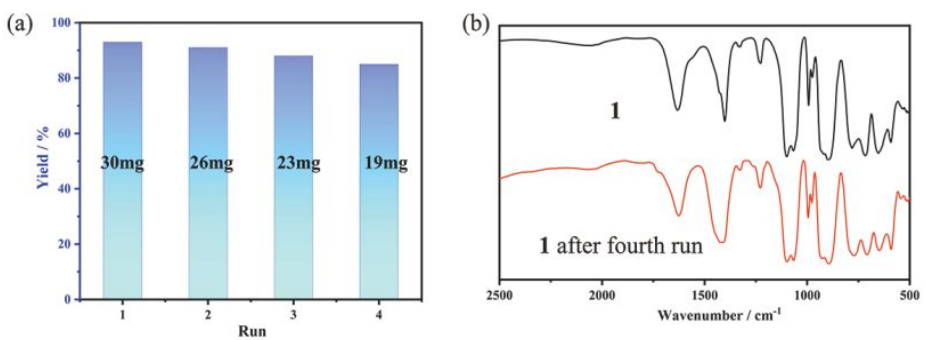

Figure S9. (a) The histogram of yield of product for reused 1; (b) The IR spectra of 1 after the fourth run. Reaction conditions: catalyst, $30 \mathrm{mg}$; ethyl cyanoacetate, $5 \mathrm{mmol}$; benzaldehyde, $7.5 \mathrm{mmol} ; \mathrm{T}, 70{ }^{\circ} \mathrm{C}$; solvent, methanol; time, $3 \mathrm{~h}$. 


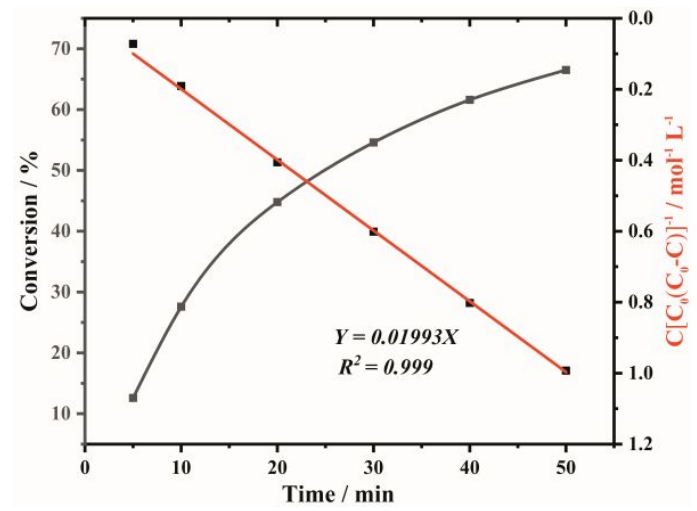

Figure S10. Kinetic profiles of the Knoevenagel condensation reaction: catalyst, $0.015 \mathrm{~mol} \%$; ethyl cyanoacetate, $5 \mathrm{mmol}$; benzaldehyde, $5 \mathrm{mmol} ; \mathrm{T}, 70^{\circ} \mathrm{C}$; solvent, methanol.

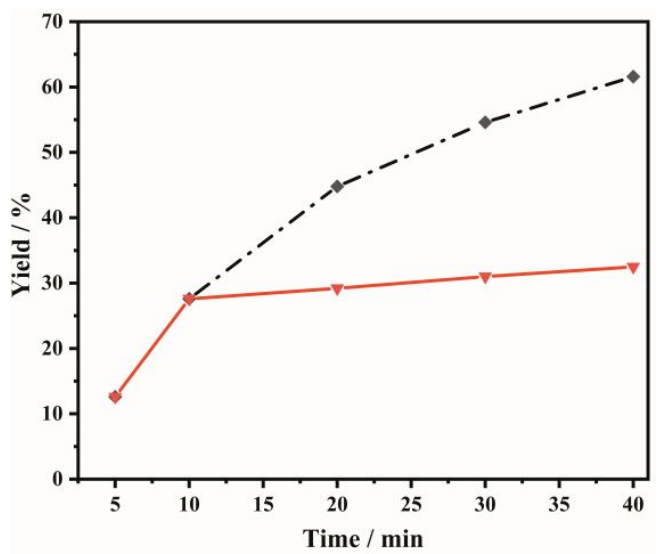

Figure S11. The results of Heterogeneous test for catalyst: catalyst, $0.015 \mathrm{~mol} \%$; ethyl cyanoacetate, $5 \mathrm{mmol}$; benzaldehyde, 7.5 mmol; $\mathrm{T}, 70^{\circ} \mathrm{C}$; solvent, methanol. 


\section{Mass spectrums for the reactants and products.}

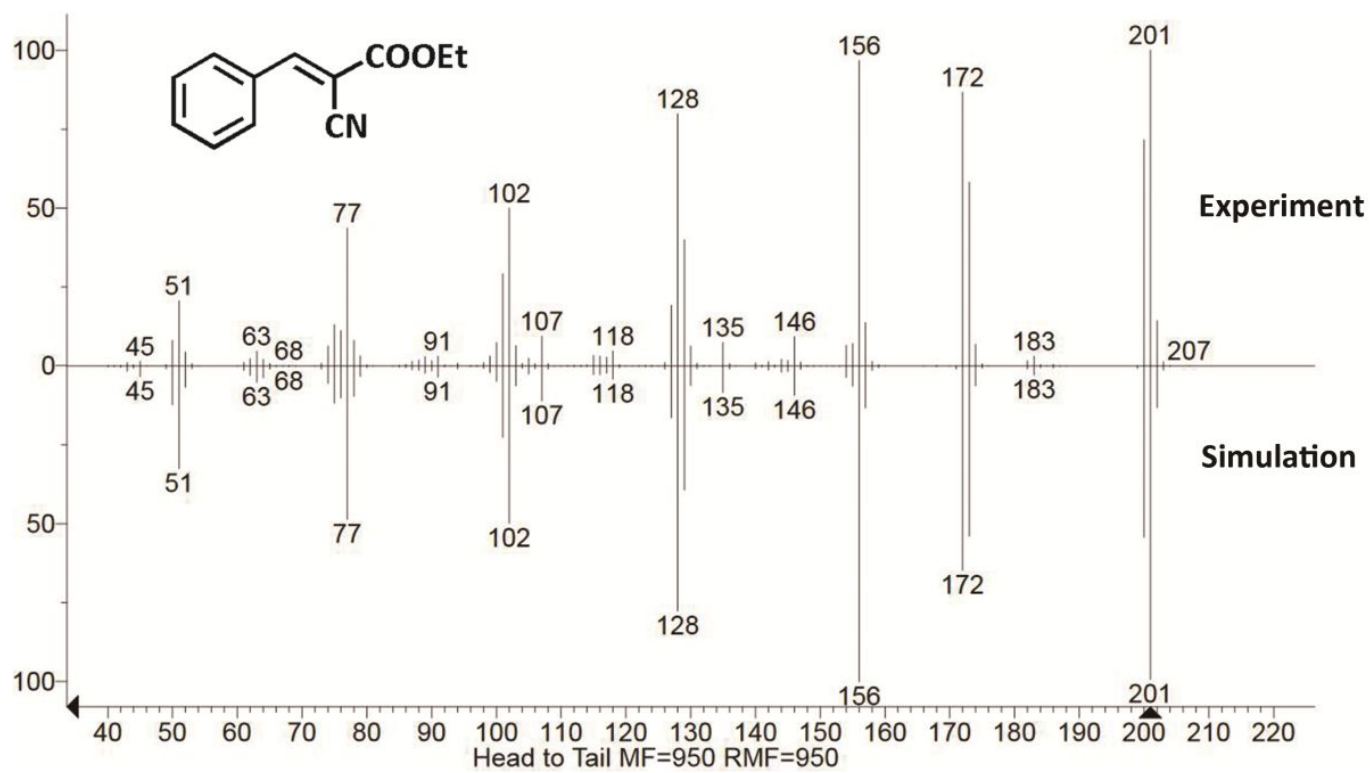

Figure S12. Mass spectrum for the product of the condensation reaction of benzaldehyde with ethyl cyanoacetate (Top, experimental; Bottom, simulation).

\section{Catalytic device}

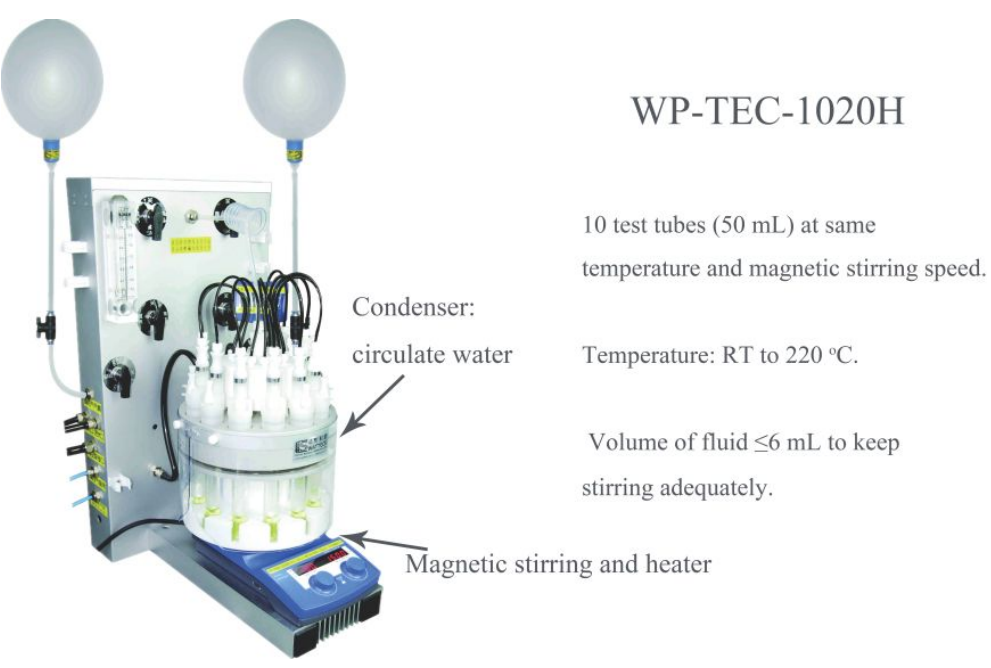

Figure S13. Catalytic device for Knoevenagel condensation with the scale of 5-7.5 mmol substrates, 9.7-29.1 mg catalyst and $2.5 \mathrm{~mL}$ solvent. 


\section{Scale-up experiment}

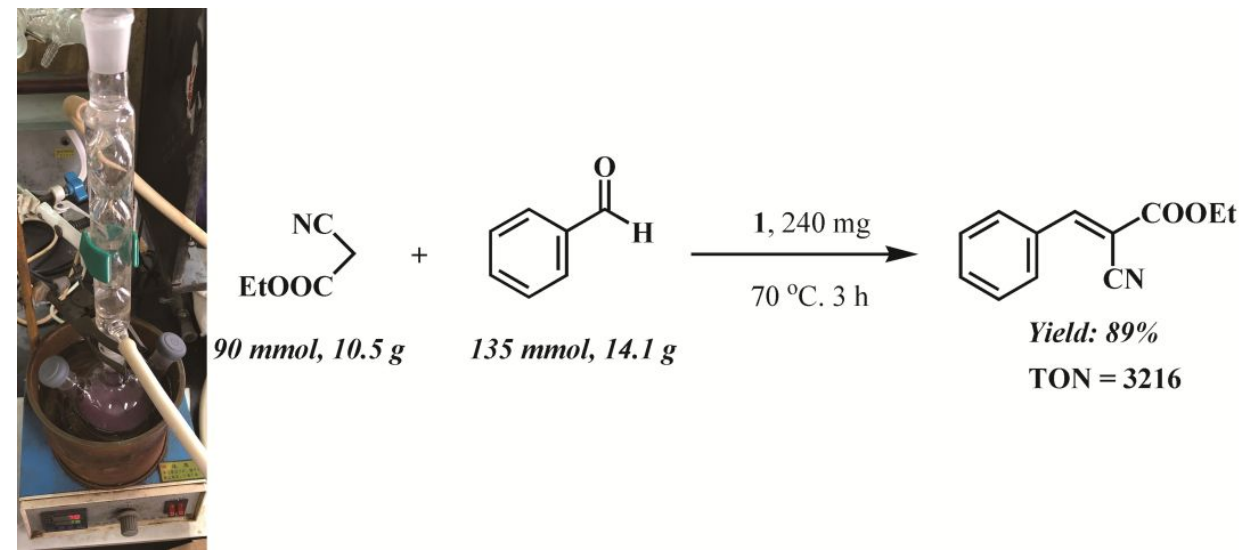

Figure S14. Scale-up experiment for Knoevenagel condensation. Reaction conditions: ethyl cyanoacetate, 90 mmol; aldehydes, $135 \mathrm{mmol}$; catalyst, $0.025 \mathrm{mmol}(240 \mathrm{mg})$; methanol, $45 \mathrm{~mL} ; 70^{\circ} \mathrm{C}$, $3 \mathrm{~h}$. 